\title{
Higher Dimensional Models of Light Majorana Neutrinos Confronted by Data
}

\author{
JoAnne L. Hewett*, 1 , 团 Probir Roy, 2 , 团 and Sourov Roy, ${ }^{3}$ 图 \\ ${ }^{1}$ Stanford Linear Acclerator Center, Menlo Park, CA 94025, USA \\ ${ }^{2}$ Department of Theoretical Physics, Tata Institute of Fundamental Research, Homi Bhabha Road, Mumbai - 400 005, India \\ ${ }^{3}$ Helsinki Institute of Physics, P.O. Box 64, FIN-00014 University of Helsinki, Finland
}

(Received November 12, 2018)

\begin{abstract}
We discuss experimental and observational constraints on certain models of higher dimensional light Majorana neutrinos. Models with flavor blind brane-bulk couplings plus three or four flavor diagonal light Majorana neutrinos on the brane, with subsequent mixing induced solely by the Kaluza-Klein tower of states, are found to be excluded by data on the oscillations of solar, atmospheric and reactor neutrinos, taken together with the WMAP upper bound on the sum of neutrino masses. Extra dimensions, if relevant to neutrino mixing, need to discriminate between neutrino flavors.
\end{abstract}

PACS numbers: 11.25.Mj, 14.60.Pq, 14.60.St

Do neutrinos have anything to do with large extra dimensions [1]? Do these extra dimensions discriminate between neutrino flavors? These questions have engaged the attention of many authors 2] during the last few years. Let us consider the simple picture of a three-brane, containing the Standard Model (SM) fields, embedded in a higher dimensional space, known as the bulk. The basic idea then is to introduce one (or more) right chiral neutrino(s), which are singlet(s) with respect to the SM gauge group, in the bulk [3]. Tiny physical masses for the active $\mathrm{SU}(2)_{\mathrm{L}}$ doublet neutrinos on the brane, that mix among themselves as well as with sterile components, then get induced. These are controlled by the size(s) of the compactified extra dimension(s) and the strength(s) of the couplings(s) between the brane and the bulk states. The bulk states expand into a Kaluza-Klein tower of states with a zero mode, as well as accompanying KK excitations of heavier sterile neutrinos with arithmetically progressing masses $M_{k}= \pm k / R, \mathrm{k}=1,2, \ldots$ with $\mathrm{R}$ being the radius of the largest extra dimension. The leakage of a propagating active neutrino into these sterile states has to be kept controllably small so as not to significantly alter the conventional oscillation probability formula [5] in the standard picture of three directly mixed active neutrinos. An immediate concern, nonetheless, is whether this picture is compatible with the pattern of neutrino masses and mixing angles implied by the latest oscillation and cosmological data. This note will address precisely this issue, focussing on the question whether the brane-bulk couplings are flavor blind or whether they are forced by all the data to have significant flavor dependence.

A relevant basic issue is whether the physical massive

\footnotetext{
${ }^{*}$ Work supported in part by the Department of Energy, Contract DE-AC03-76SF00515

${ }^{\dagger}$ Electronic address: hewett@SLAC.Stanford.EDU

‡Electronic address: probir@theory.tifr.res.in

$\S$ Electronic address: roy@pcu.helsinki.fi
}

neutrinos are Dirac or Majorana particles. There is a relatively straightforward picture in the former case. One combination of the bulk neutrinos now becomes the right chiral partner of an active neutrino on the brane. Together they acquire a Dirac mass $m_{D} \sim h v M_{F} / M_{P l}$, where $v$ is the electroweak vacuum expectation value (VEV), $h$ is a Yukawa coupling strength (controlling the interaction between the brane and bulk states) which is of order unity or less, $M_{P l}$ the reduced Planck mass in $(1+3)$ dimensions whereas $M_{F}$ is the higher dimensional fundamental scale, proposedly in the multi-TeV range. This scenario was given a thorough phenomenological examination in Ref. [6] with the conclusion that it is consistent with all extant experimental and observational constraints, provided $R^{-1}>0.24 \mathrm{eV}$. However, despite providing a natural explanation of the smallness of neutrino masses, this approach sheds no light on the observed pattern of neutrino mixing, nor on the hierarchy - if any - between the neutrino masses. These supposedly arise from the unknown structure of the Yukawa coupling matrix $h$ in generation space. Any observed pattern of mixing and mass hierarchy among the neutrinos can be accommodated by this unknown structure, with the only constraint arising from the strict absence of neutrinoless double $\beta$-decay. A simple generalization of this picture with heavy right chiral Majorana neutrinos in the bulk leads to light physical Majorana neutrinos via the seesaw mechanism. There, one also needs to invoke (unknown) flavor dependent brane-bulk couplings.

There are higher dimensional scenarios [7, 8, 9, 10] with a somewhat opposite approach. These assume tiny flavor diagonal Majorana masses for left chiral neutrinos on the brane without explaining their smallness, but invoke extra dimensions to generate their mixing pattern. Here the higher dimensional KK modes induce a seesaw leading to light physical Majorana neutrinos with flavor mixing. However, this mixing pattern is strongly constrained. Suppose [1] that (1) no flavor dependence is assumed in the couplings between the brane and bulk 
states and (2) flavor mixing is not allowed on the brane so that the brane Majorana neutrinos are kept flavor diagonal 7, 8, 9, 10]. Then, our observation is that the Dienes-Sarcevic model 7, 12], with three flavor diagonal neutrinos on the brane, is ruled out by the data. The allowed parameter space actually gets quite constrained [8] even when an arbitrary flavor dependence is included in the brane-bulk couplings. We also find that a version of the model, given in Ref. 9] with four flavor diagonal brane Majorana neutrinos and which has flavor blind brane-bulk couplings, is excluded.

One attractive feature of the D-S model [7] is the abil- ity to provide a theory of neutrino mixing instead of just ascribing it to an unknown flavor dependence of the brane-bulk couplings. The intrinsic flavor diagonal Majorana masses on the brane for the active neutrinos $\nu_{f}(f$ $=1,2,3)$ are taken to be $m_{f}$, while the brane-bulk linkage is characterized by a universal mass $m$ appearing in all brane-bulk off-diagonal elements of the full neutrino Majorana mass matrix $\mathcal{M}$. Compactification to four dimensions yields the zero mode as well as to a tower of sterile neutrino KK states, as mentioned above. After the imposition of appropriate orbifold conditions, the full neutrino Majorana mass matrix in the D-S model reads

$$
\mathcal{M}=\left(\begin{array}{ccccccccc}
m_{1} & 0 & 0 & m & m & m & m & m & \ldots \\
0 & m_{2} & 0 & m & m & m & m & m & \ldots \\
0 & 0 & m_{3} & m & m & m & m & m & \ldots \\
m & m & m & 0 & 0 & 0 & 0 & 0 & \cdots \\
m & m & m & 0 & 1 / R & 0 & 0 & 0 & \ldots \\
m & m & m & 0 & 0 & -1 / R & 0 & 0 & \ldots \\
m & m & m & 0 & 0 & 0 & 2 / R & 0 & \ldots \\
m & m & m & 0 & 0 & 0 & 0 & -2 / R & \ldots \\
\vdots & \vdots & \vdots & \vdots & \vdots & \vdots & \vdots & \vdots & \ddots
\end{array}\right)
$$

In consequence of (10), the active neutrinos on the brane mix both among themselves and with the sterile KK states. Thus they undergo flavor oscillations on propagation. Let $U$ be the unitary matrix which acts on mass eigenstate neutrino fields to yield flavor eigenstate neutrino fields. $U^{T} \mathcal{M} U$ is then diagonal. The matrix $U$ therefore is given by the inverse of the matrix of eigenvectors of $\mathcal{M}$ and is computable as such. The time-averaged probability $\overline{P_{f \rightarrow f^{\prime}}}$ that $\left|\nu_{f}\right\rangle$ oscillates into $\left|\nu_{f^{\prime}}\right\rangle$, with both $f$ and $f^{\prime}$ being flavor indices on the brane, is given by [2, 5, 7]

$$
\overline{P_{f \rightarrow f^{\prime}}}=\delta_{f f^{\prime}}-2 \sum_{i=2}^{N} \sum_{j=1}^{i-1} \operatorname{Re}\left[U_{f i} U_{f^{\prime} i}^{*} U_{f j}^{*} U_{f^{\prime} j}\right],
$$

where (2), i and $\mathrm{j}$ are neutrino mass eigenstate indices.

Let us now discuss the experimental results for the physical neutrino mass squared differences and the average neutrino oscillation probabilities. The atmospheric neutrino data yield [13, 14]

$$
\begin{aligned}
1.3 \times 10^{-3} \mathrm{eV}^{2} & <\Delta m_{\mathrm{atm}}^{2}<3.2 \times 10^{-3} \mathrm{eV}^{2}, \\
0.9 & <\sin ^{2} 2 \theta_{\mathrm{atm}} \leq 1 .
\end{aligned}
$$

The combined solar neutrino observations plus the KamLAND data imply [15, 16, 17]

$$
\begin{aligned}
6.1 \times 10^{-5} \mathrm{eV}^{2} & <\Delta m_{\mathrm{sol}}^{2}<9.0 \times 10^{-5} \mathrm{eV}^{2} \\
0.71 & <\sin ^{2} 2 \theta_{\text {sol }}<0.91
\end{aligned}
$$

The data 18] from the CHOOZ reactor require that 19]

$$
\sin ^{2} 2 \theta_{13} \leq 0.26 \text {. }
$$

In an effectively two flavor oscillation with a mixing angle $\phi$, the time-averaged oscillation probability is $\frac{1}{2} \sin ^{2} 2 \phi$. We can therefore use the estimates

$$
\begin{aligned}
0.45 & <\overline{P_{\mathrm{atm}}}<0.5, \\
0.355 & <\overline{P_{\mathrm{sol}}}<0.455, \\
\overline{P_{13}} & \leq 0.13
\end{aligned}
$$

in our analysis 20]. Finally, the recent WMAP result puts 21] an upper bound on the sum of the physical masses of stable light neutrinos that mix among one another, namely

$$
\sum_{i} m_{i}<0.71 \mathrm{eV}
$$

A constraint, therefore, on any model is that there must be at least three light neutrinos with the sum of their masses not exceeding $0.71 \mathrm{eV}$.

It has been argued by Pierce and Murayama 22 that the WMAP upper bound should include any light sterile neutrino that mixes with the active ones. This mixing is, of course, restricted to be rather small by the LEP constraint on invisible $\mathrm{Z}$ decay. A wide window for the existence of a sterile neutrino is nonetheless allowed by the WMAP results. Suppose the active fraction of the sterile neutrino is $\epsilon$ so that $\epsilon^{2} \sim \sin ^{2}\left(2 \theta_{m i x}\right)$, yielding $\epsilon \leq 10^{-1}$. 
Such a neutrino would have decoupled in the early universe at a temperature $\mathrm{T}$ where $G_{F}^{2} T^{5} \epsilon^{2} \sim T^{2} M_{P l}^{-1}$. This temperature simply must be less than that of the QCD phase transition in order to avoid the dilution of those neutrinos by the entropy produced at this transition. Thus $\epsilon$ must lie in the the range $10^{-6}<\epsilon<10^{-1}$. This means that in most regions of the parameter space of the models examined here, the summation in (II) includes the contribution of light sterile neutrinos.

There are five parameters in the Dienes-Sarcevic (DS) model : the input Majorana masses $m_{1,2,3}$ for the three neutrinos on the brane, the universal brane-bulk off-diagonal coupling $m$ and the radius of the compact extra dimension $R$. For the present study we can set all mass scales in $\mathrm{eV}$ and $R$ in $\mathrm{eV}^{-1}$. Let us also comment on the infinite number of sterile neutrinos in the model. There are, of course, certain limiting regions of the D-S parameter space when all the sterile neutrinos are sufficiently heavy to be effectively decoupled from the phenomena leading to (3) - (11). There are other regions where one or more sterile states can become part of the light neutrino spectrum. The existence of such light sterile neutrinos is already somewhat disfavored 23] by analyses of existing neutrino data. Though the situation is far from definite, results from the ongoing mini-Boone experiment are expected to provide a clearer conclusion. At the moment, several light sterile neutrinos can still be accommodated.

We now come to a numerical investigation of the D-S model in the five-dimensional parameter space $\left(m_{1}, m_{2}\right.$, $\left.m_{3}, m, R^{-1}\right)$. An initial requirement is that there must be at least three light neutrinos with the sum of their masses obeying (11). Thus those regions of the parameter space, which are not consistent with this constraint, are immediately excluded. We attribute the masses $\mu_{1,2,3}$ to the physical light neutrinos, always ordered [24] such that $\mu_{3}>\mu_{2}>\mu_{1}$. The conditions (3) and (5) then apply on the pertinent differences of squared mass eigenvalues. After employing these conditions, we have made a complete scan of the parameter space. Our quest is to determine if there is any region in which all the other constraints, i.e. (7) to (11), can be satisfied. Our answer is in the negative, i.e. there is no point in the parameter space where all the four conditions can hold simultaneously. As will be shown below, there are parts of the parameter space where (18), (10) and (11) can be simultaneously satisfied. Similarly, there are other regions where the conditions of (9), (10) and (11) can be met. But these two sets of points do not overlap. Our conclusion is that the D-S model is ruled out by the data.

From practical considerations in making our scan, we have taken the number of contributing KK states $\mathrm{N}$ in (2) to be finite though there actually are an infinite number of states in the KK tower. We choose $\mathrm{N}=40$ but have checked that varying this truncation affects our results negligibly. Furthermore, the choice of parameters in our numerical scanning was dictated by the eigenvalues of the mass-matrix (1), as described below. Consider, for the moment, the possibility of flavor dependent couplings for the brane neutrinos, leading to off-diagonal elements $m_{i}^{\prime}$ of $\mathcal{M}$. The eigenvalues of $\mathcal{M}$ are then determined by the characteristic equation

$$
\frac{1}{\pi} \tan (\pi \lambda)=\sum_{i=1}^{3} \frac{m_{i}^{\prime 2} R^{2}}{\lambda-m_{i} R} .
$$

Here, $\lambda=\mu R$ and $\mu$ represents the eigenvalues. Let us also define $d^{2} \equiv R^{2}\left(\sum_{i=1}^{3}{m_{i}^{\prime}}^{2}\right)$ so that $m_{i}^{\prime} R=d e_{i}$, where $\sum_{i=1}^{3} e_{i}^{2}=1$. The two zeros of

$$
r(\lambda) \equiv \sum_{i=1}^{3} \frac{e_{i}^{2}}{\lambda-m_{i} R}
$$

are given in the limit $m_{1}^{\prime}=m_{2}^{\prime}=m_{3}^{\prime}=m$ (i.e., $e_{1}=$ $e_{2}=e_{3}=e$ ) by

$$
\begin{aligned}
\frac{\lambda_{b, c}}{R} & =\mu_{b, c}=\frac{1}{2}(p \mp c), \\
p & =2 e^{2}\left(m_{1}+m_{2}+m_{3}\right), \\
c & =\left[p^{2}-4 e^{2}\left(m_{2} m_{3}+m_{3} m_{1}+m_{1} m_{2}\right)\right]^{1 / 2} .
\end{aligned}
$$

It has been shown in Ref. 8] that, as $d$ increases, two of the eigenvalues move towards $\mu_{b}$ and $\mu_{c}$ whereas the other eigenvalues move towards half-integral values times $R^{-1}$. For $d \gg 1$, the two eigenvalues sit at $\mu_{b}$ and $\mu_{c}$ (independent of $R$ ), while the rest are fixed at those halfinteger values times $R^{-1}$. We have verified this property numerically. We have thus not included large values of $m(m R=d e)$ in our numerical scanning of the parameter space, since that would not allow for two different mass squared differences as required to explain both the solar and the atmospheric neutrino deficits. The other significant constraint on the range of $m_{1,2,3}$ is (11). Let us now consider variations in the radius of compactification $R$. One should note first that, so long as the dimensionless products $m_{1} R, m_{2} R, m_{3} R$ and $m R$ remain the same, one would get the same time-averaged oscillation probabilities. We have further checked that, for a fixed set of $\left(m_{1}, m_{2}, m_{3}\right.$ and $\left.m\right)$, our conclusions do not change with the variation in $R$ permitted by the constraints given in (3), (51) and (11). This way $R$ can be seen to be constrained in the range aproximately given by $10^{-6} \mathrm{eV}^{-1} \lesssim R \lesssim 10 \mathrm{eV}^{-1}$.

In our convention [24], $\Delta m_{\mathrm{sol}}^{2}=\mu_{2}^{2}-\mu_{1}^{2}$ and $\Delta m_{\mathrm{atm}}^{2}=$ $\mu_{3}^{2}-\mu_{2}^{2}$, for a normal ordering of neutrino masses, while $\Delta m_{\text {sol }}^{2}=\mu_{3}^{2}-\mu_{2}^{2}$ and $\Delta m_{\text {atm }}^{2}=\mu_{2}^{2}-\mu_{1}^{2}$ in the case of an inverted ordering. Allowed regions, in the $m_{1}-m_{3}$ plane for the choice of $R^{-1}=1 \mathrm{eV}$, are shown in Figs. 1 (a) and (b). The incompatibility of the D-S model with the presently known facts from neutrino oscillation and cosmological data is made evident by these figures. In both figures $m_{1}, m_{2}$ and $m_{3}$ are taken to vary between $0.001 \mathrm{eV}$ and $0.31 \mathrm{eV}$, whereas $m$ is varied between 0.001 $\mathrm{eV}$ and $0.075 \mathrm{eV}$. Though, in the inverted case, a few 

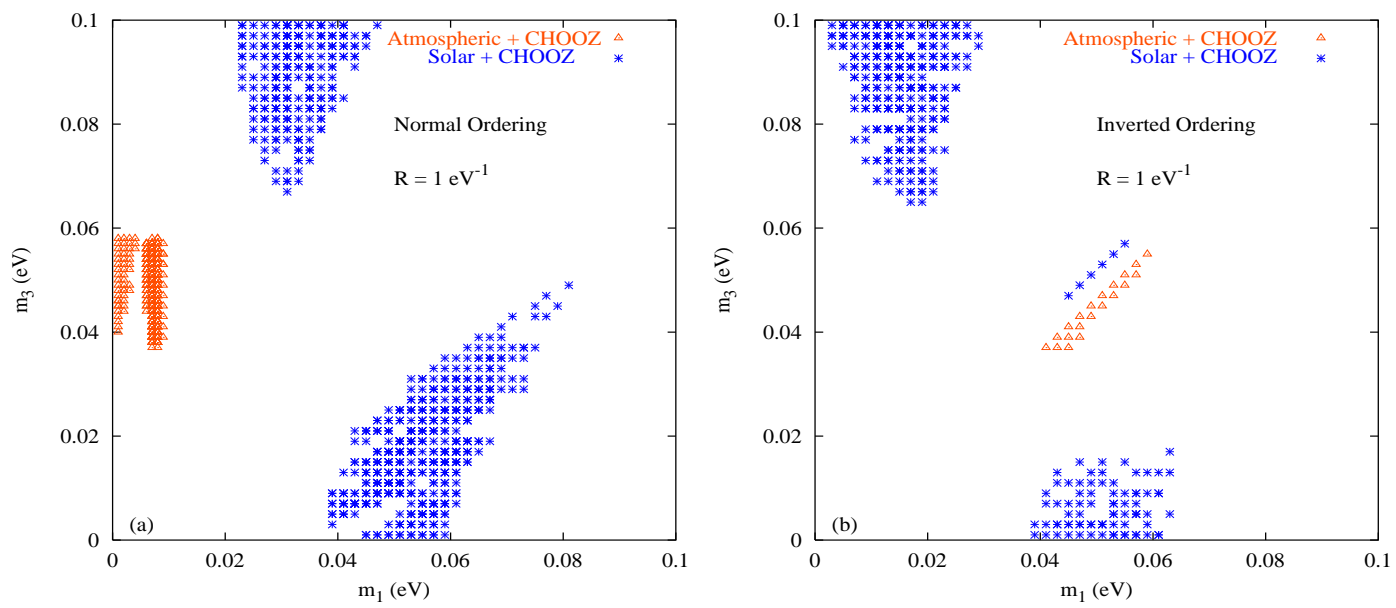

FIG. 1: Allowed regions in the $m_{1}$ and $m_{3}$ plane for (a) the normal ordering and (b) the inverted ordering of neutrino masses. Choices of parameters are described in the text.

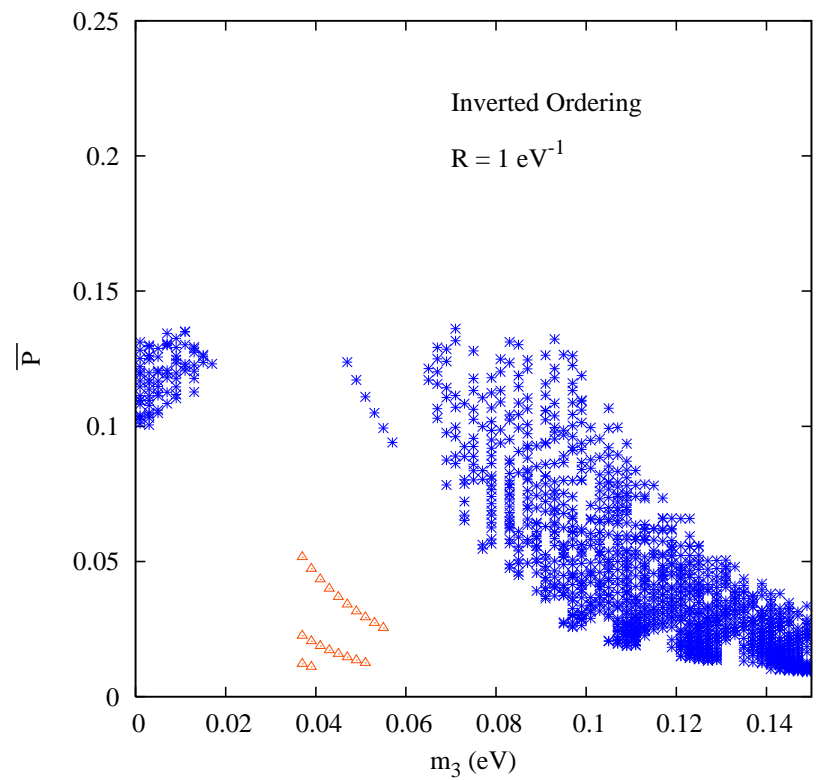

FIG. 2: Time-averaged oscillation probability (a) $\overline{P_{a t m}}$ (in Blue/star) and (b) $\overline{P_{\text {sol }}}$ (in orange/triangle) for the inverted ordering of neutrino masses as a function of $m_{3}$. Only those points are considered here which satisfy for (a) the Solar+CHOOZ and for (b) Atmospheric + CHOOZ constraints.

points satisfying the conditions of (9), (10) do lie close to the points satisfying (8), (10), in fact there is not a single point in the five-dimensional parameter space of $\left(m_{1}, m_{2}, m_{3}, m, R^{-1}\right)$ which is compatible with all the constraints. To further convince the reader, we display in Fig. 2 the time-averaged oscillation probability (a) $\overline{P_{a t m}}$ and (b) $\overline{P_{\text {sol }}}$ as a function of $m_{3}$ in the inverted case for all points shown in Fig 1 Clearly, there is no consistent region of parameter space. We have checked that this holds as $\mathrm{R}$ is varied in the range given above.

Next, we consider the model of Lam [9] which requires a sterile neutrino (with a Majorana mass $m_{4}$ ) on the brane in addition to three active neutrinos. In the limit of large coupling between the brane neutrinos and the bulk (i.e., $\left.d \gg m_{i} R, 1\right)$ one can have three "isolated" eigenvalues which can give rise to two different $\Delta m^{2}$, whereas the other eigenvalues sit at half-integer values. Following Lam, we also arrange the Majorana masses of the brane neutrinos in such a way that

$$
0<m_{1}<\mu_{1}<m_{2}<\mu_{2}<m_{3}<\mu_{3}<m_{4},
$$

where $\mu_{i}$ are the isolated eigenvalues. Furthermore, we assume that the $m_{i} R$ do not have integral or half-integral values. We take a flavor-blind brane-bulk coupling $m$ as in the earlier scenario. After a thourough scan in the parameter space with the above mentioned constraint in (15), we find that although in some part of the parameter space it is possible to satisfy the constraints in (3) and (5), there is no region where both the constraints in (8) and (9) can be satisfied either with a normal or with an inverted ordering of the neutrino masses.

In summary, we are able to exclude the Dienes-Sarcevic model [] and that version of Lam's model [9] which has flavor blind off-diagonal mass terms, by using all neutrino oscillation data and the WMAP constraint on the sum of neutrino masses. Extra dimensional models of neutrino 
masses and mixing angles need flavor dependent branebulk couplings.

P.R. and S.R. acknowledge the hospitality of the Theory Group at SLAC where this work was initiated. We thank S. Choubey, S. Goswami, A.S. Joshipura and H-
J. He for their helpful comments. The research of S.R. was supported by the Academy of Finland (Project number 48787). S.R. would also like to thank the Lady Davis Fellowship Trust in Technion, Israel, for financial support during the initial stages of this work.
[1] N. Arkani-Hamed, S. Dimopoulos and G.R. Dvali, Phys. Lett. B429, 263 (1998); I. Antoniadis, N. Arkani-Hamed, S. Dimopoulos and G.R. Dvali, ibid. B436, 257 (1998); L. Randall and R. Sundrum, Phys. Rev. Lett. 83, 3370 (1999); ibid. 4690 (1999).

[2] K.R. Dienes, E. Dudas and T. Gherghetta, Nucl. Phys. B557, 25 (1999); G.R. Dvali and A.Y. Smirnov, Nucl. Phys. B563, 63 (1999); A.E. Faraggi and M. Pospelov, Phys. Lett. B458, 237 (1999), J. Maalampi, V. Sipilainen and I. Vilja, Phys. Lett. B512, 91 (2001); Phys. Rev. D67, 113005 (2003); R.N. Mohapatra and A. PérezLorenzana, Nucl. Phys. B576, 466 (2000); ibid. B593, 451 (2001); R. Barbieri, P. Creminelli and A. Strumia, Nucl. Phys. B585, 28 (2000); A. Lukas, P. Ramond, A. Romanino and G.G. Ross, Phys. Lett. B498, 230 (2001), D.O. Caldwell, R.N. Mohapatra, and S.J. Yellin, Phys. Rev. D64, 073001 (2001); N. Cosme, J.-M. Frère, Y. Gouverneur, F.-S. Ling, D. Monderen and V. Van Elewyck, Phys. Rev. D63, 113008 (2001); K. Agashe and G.H. Wu, Phys. Lett. B498, 230 (2001); A. Ioannisian and A. Pilaftsis, Phys. Rev. D62, 066001 (2000); G. Bhattacharyya, H.V. Klapdor-Kleingrothaus, H. Päs and A. Pilaftsis, Phys. Rev. D67, 113001 (2003); A. Dighe and A.S. Joshipura, Phys. Rev. D64, 073012 (2001), N. Arkani-Hamed, S. Dimopoulos, G.R. Dvali and J. MarchRussel, Phys. Rev. D65, 024032 (2002); T. Appelquist, B. Dobrescu, E. Ponton and H. Yee, Phys. Rev. D65, 105019 (2002).

[3] There are more complicated models consisting of right chiral neutrinos on the brane and/or left chiral ones in the bulk with warped geometric configurations [4], but the use of flavor dependent brane-bulk couplings to explain the observed pattern of neutrino mixing is quite generic in these.

[4] Y. Grossman and M. Neubert, Phys. Lett. B474, 361 (2000); G. C. McLaughlin and J.N. Ng, Phys. Lett. B493, 88 (2000); S.J. Huber and Q. Shafi, J. Phys. G29, 1807 (2003) and Phys. Lett. B583, 293 (2004).

[5] S.M. Bilenky and S.T. Petkov, Rev. Mod. Phys. 59, 671 (1989).

[6] H. Davoudiasl, P. Langacker and M. Perelstein, Phys. Rev. D65, 105015 (2002).

[7] K.R. Dienes and I. Sarcevic, Phys. Lett. B500, 133 (2001).

[8] C.S. Lam and J.N. Ng, Phys. Rev. D64, 113006 (2001).

[9] C.S. Lam, Phys. Rev. D65, 053009 (2002).

[10] Q-H. Cao, S. Gopalakrishna and C.P. Yuan, hep-ph/0312339

[11] Thus the entire flavor mixing among the light physical neutrinos is induced by the KK modes.

[12] An extension of the model of Ref. [7] with three different off-diagonal mass elements, was investigated in the limit of their very large values by Lam and $\mathrm{Ng}$ [8] and shown to be incompatible with the data. Our study, however, covers the entire parameter space of the Dienes-Sarcevic model [7] with a uniform off-diagonal mass.

[13] We use $90 \%$ C.L. limits throughout.

[14] M. Shiozawa, super-Kamiokande coll. in XX Intl. Conf. Neutrino Physics and Astrophysics, Munich, May 2002 (http://neutrino2002.ph.tum.de); S. Fukuda et al. superKamiokande coll. Phys. Rev. Lett. 81, 1562 (1998); ibid. 85, 3999 (2000).

[15] Q.R. Ahmad et al., SNO Coll., Phys. Rev. Lett. 87, 071301 (2001); ibid. 89, 011301 (2002); S. Fukuda et al., super-Kamiokande coll. Phys. Lett. B539, 179 (2002); B.T. Cleveland et al., Astrophys J. 496, 505 (1998); W. Hampelet et al. Phys. Lett. B447, 127 (1999); J.N. Abduradutov et al. JETP 95, 181 (2002); Y. Fukuda et al., Phys. Rev. Lett. 77, 1683 (1996); M.H. Ahn et al., Phys. Rev. Lett. 90, 041801 (2003).

[16] S.N. Ahmed et al., nucl-ex/0309004

[17] M.C. Gonzalez-Garcia and C. Pena-Garay, Phys. Rev. D68, 093003 (2003).

[18] M. Apollonio et al., Phys. Lett. B466, 415 (1999).

[19] M. Maltoni, T. Schwetz, M.A. Tortola and J.W.F. Valle, Phys. Rev. D68, 113010 (2003); G.L. Fogli, E. Lisi, A. Marrone, D. Montanind, A. Palazzo and A.M. Rotunno, Phys. Rev. D69, 017301 (2004); A. Bandyopadhyay, S. Choubey, S. Goswami, S.T. Petkov and D.P. Roy, Phys. Lett. B583, 134 (2004).

[20] Our conclusions follow just from these averaged probabilities without any need to use the detailed shape of the corresponding distributions.

[21] D.N. Spergel et al., Astrophys. J. Suppl. 148, 175(2003).

[22] A. Pierce and H. Murayama, Phys. Lett. B581, 218 (2004); G. Bhattacharyya, H. Päs, L. Song, and T. Weiler, Phys. Lett. B564, 175 (2003); P. Crotty, J. Lesgourgues and S. Pastor, hep-ph/0402049

[23] M. Maltoni, T. Schwetz, M.A. Tortola and J.W.F. Valle, Nucl. Phys. Proc. Suppl. 114, 203 (2003); M.C. Gonzalez-Garcia, M. Maltoni and C. Pena-Garay, Phys. Rev. D64, 093001 (2001).

[24] This convention of always keeping $\mu_{3}>\mu_{2}>\mu_{1}$, which is different from the more common PMNS convention of attributing $\mu_{1,2,3}$ to $\left|\nu_{e, \mu, \tau}\right\rangle$ in the 'no-mixing' limit $U \rightarrow I$, has been followed for search convenience and is inessential to the derivation of the final conclusion. 